\title{
Classifying Skin Neoplasms with Infrared Thermal Images
}

\author{
by C. Magalhaes*, R. Vardasca **, J. Mendes** \\ * Faculdade de Engenharia, Universidade do Porto, Rua Dr. Roberto Frias S/N 4200-465 Porto, Portugal \\ ** LABIOMEP, INEGI-LAETA, Faculdade de Engenharia, Universidade do Porto, Rua Dr. Roberto Frias $S / N$ \\ 4200-465 Porto, Portugal, rvardasca@fe.up.pt
}

\begin{abstract}
The cases of benign and malignant skin neoplasms have been rising over the last decades. They are an esthetical and health threat and require prompt attention, the current diagnostic methods are the biopsy (expensive and invasive) and the dermatoscopy (experience dependent), so new objective methods are needed. The growth of neoplasms is influenced by blood flow, influencing, thereby, skin temperature. Infrared thermal images were taken from 85 patients at the cancer hospital, being analysed and classified with different intelligent data methods. The method that outperformed the other was the k-Nearest Neighbour with $60 \%$ accuracy.
\end{abstract}

\section{Introduction}

According to the 2012 statistics, in Europe there is an incidence rate for melanomas of 11.1 per 100000 population. Comprehensive statistics on the occurrence of non-melanoma skin neoplasia are scarce but is well acknowledged that its number exceeds largely those of melanoma [1].

The increased number of skin cancer cases has been connected to excessive exposure to ultraviolet radiation, particularly during child and young adult years. Poor recurrent lifestyle choices, e.g., use of tanning beds and non-use of sunscreen, of younger individuals, indicates that these values are likely to rise [2]. Hence, special attention is required.

The available diagnostic methods for skin neoplasia are dermatoscopy, used in the evaluation of melanocytic lesions, and biopsy, mainly performed when the malignancy of a skin tumor is doubtful. Both methods present disadvantages, being the first a subjective technique, due to its' dependence on physician experience, and the second an invasive and costly procedure that is frequently associated with increased patient stress and unaesthetic scars [3]. Thus, it is important to explore new methodologies that provide a prompt diagnosis without the disadvantages encountered in common strategies.

Infrared thermal imaging (IRT) is able to monitor the temperature variations of a given skin area, through the use of infrared thermal cameras. This imaging technique can be implemented actively - dynamic thermography - with the application of a cold or hot stimulus to disturb the thermal balance, or passively - static thermography - when thermograms are acquired without any temperature stress [4]. The emitted skin heat is highly dependent on the peripheral blood flow, an anatomical characteristic that is largely affected when a physiological alteration, such as the growth of a skin neoplasm, takes place [5]. Considering that malignant tumors present metabolic activities that exceed the ones of benign lesions, different temperature disturbances should be expected for different neoplasia, namely, hyperthemic patterns for the first and hypothermic for the second. This factor associated with the various advantages of IRT, e. g., non-invasiveness, contactless, inexpensive, fast and non-ionizing [6], have made authors explore the use of IRT for skin cancer diagnosis over the last years [7].

The strategy adopted for image analysis and processing is the key to retrieved temperature values and/or parameters that allow a distinctive characterization of the skin lesion under evaluation. Artificial intelligence computational classification methods are frequently associated with this process and have been employed in medical IRT since 2002 with several applications, being the k-Nearest Neighbor (k-NN) the technique that provided better results, followed by Support Vector Machines (SVM) and Artificial Neural Networks (ANN) [8].

In this study a MATLAB application for the collection of temperature values, of a given skin area, was constructed with the goal of attaining the temperature profile of different skin neoplasm types, through thermal images acquired with steadystate thermography, for posterior classification.

\section{Methodology}

The collection of thermal images was performed at Instituto Português de Oncologia do Porto Francisco Gentil, EPE, cancer dedicated hospital, being the research project approved by the hospital ethical committee. A total of 85 skin oncological patients participated in this study after reading the Participant Information sheet and signing the Informed Consent. The lesions were present in several locations such as the face, upper limbs, back, thorax and lower limbs.

The data collection followed the international guidelines [9], [10] using a 10 minute acclimatization period and the room being acclimatized in a ambient temperature around $22^{\circ} \mathrm{C}$ with a relative humidity of $<50 \%$. The Infrared camera used to acquire the thermograms was the FLIR E60sc, with a FPA sensor size of 320x240, a Noise-Equivalent 
Temperature Difference (NETD) of $50 \mathrm{mK}$ at $30^{\circ} \mathrm{C}$ and a measurement uncertainty of $\pm 2 \%$ of the overall reading range. The population studied included 85 skin lesions: 35 Basal Cell Carcinomas (BCC), 26 Melanomas, 10 Melanocytic Nevus (MN), 7 Squamous Cell Carcinoma (SCC), 2 Seborrheic Keratosis (SK), 2 Actinic Keratosis (AK), 1 Atypical Nevus (AN), 1 Merkel Cell Carcinoma (MCC) and 1 Chondritis. All the neoplasia diagnoses were confirmed by a dermatologist and histopathologic results, i.e., biopsy.

The thermal images were opened in the FLIR ThermaCAM Researcher Pro 2.10 software to convert the temperature matrix format to MATLAB files. A MALTAB application was developed to read the loaded temperature matrix and display the image (Figure 1). A user input was requested to draw a square encompassing the entire lesion. The selected area was then dimensioned to a 40 by 40 -pixel size (Figure 2A) and the temperature values of the 2 cross-sections of the diagonals over the normalized square were multiplied (Figure 2B) to obtain the characteristic thermal profiles (Figure $2 \mathrm{C}$ ) of the lesion under evaluation.

These curves were primarily used for statistical calculations, were the average temperature profile was obtained, first, by groups of benign - Melanocytic Nevus, Seborrheic Keratosis, AK and Atypical Nevus - and malignant - Melanoma, BCC, SCC and Merkel Cell Carcinoma - neoplasms and second, for each type of skin tumour, separately. All 85 skin lesions were included in this analysis.

Then, the profiles were applied as inputs to the advanced data classification methods: Multilayer Percepton, a feedforward ANN model of interconnected nodes that maps, in a non-linear way, the connections between the input and the output data [11], a more practical Support Vector Machine (SVM) algorithm that simplifies the calculations performed when the number of variables is higher [12], and Instance Based for K-Nearest neighbour (k-NN), that assigns each instance to a given group according to the similarity with the majority of its neighbour [13]. The classification methods were run into the open-source suite of machine learning software Weka (https://www.cs.waikato.ac.nz/ml/weka). The standard values of WEKA parameters were used. Considering the small number of samples for Seborrheic Keratosis, AK, Atypical Nevus, Merkel Cell Carcinoma and Chondritis, in this classification stage only 50 lesion profiles were selected: 26 BCC, 14 Melanomas, 6 Melanocytic Nevus and 4 SCC. The samples were randomly divided into training and test sets of 25 instances, each.

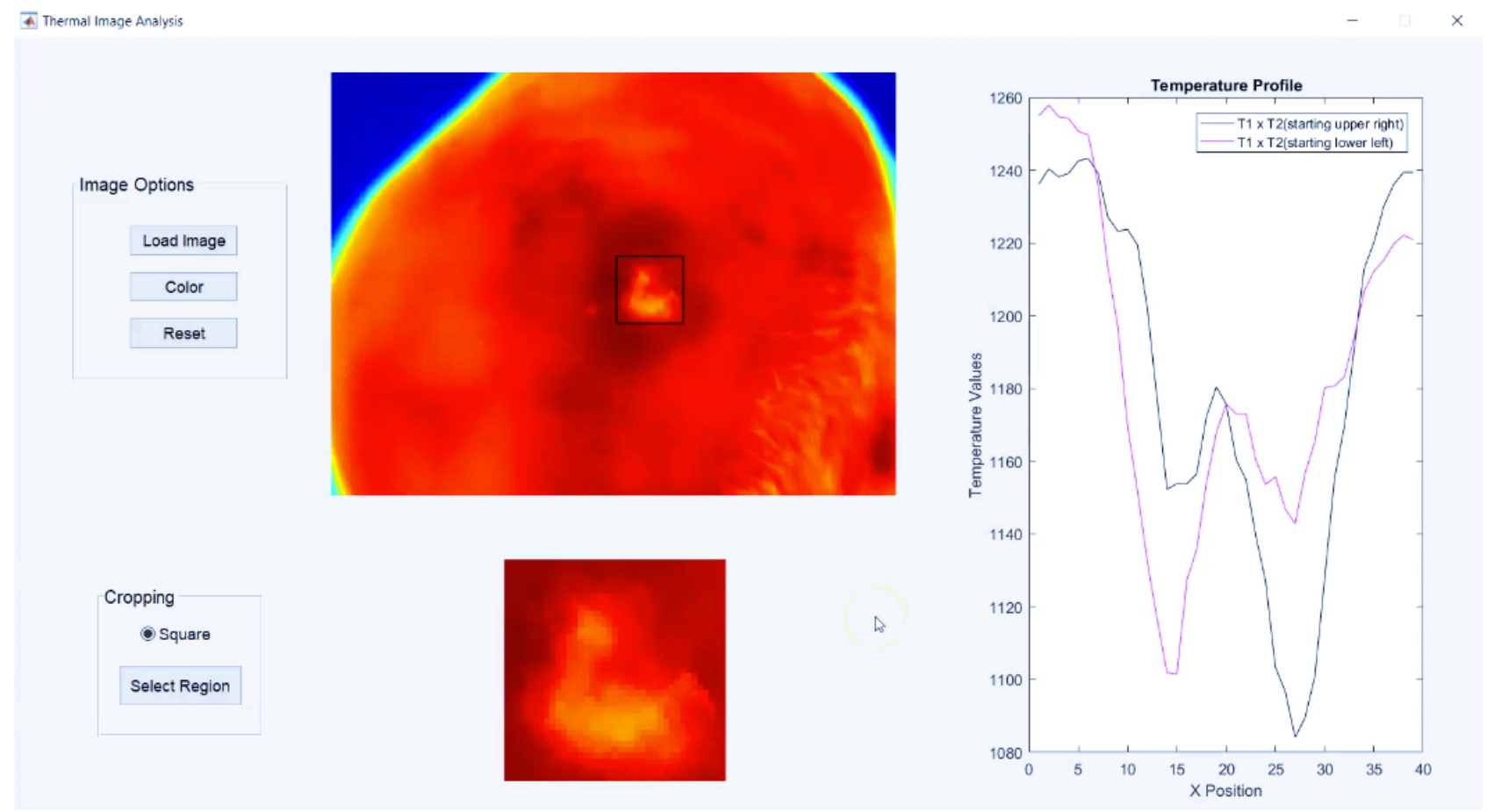

Fig. 1. The Graphic User Interface to process the thermal images of the skin neoplasms developed on Matlab. 
A

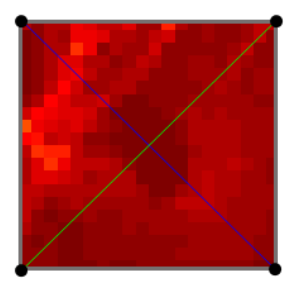

B

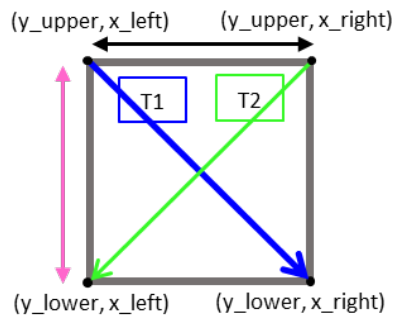

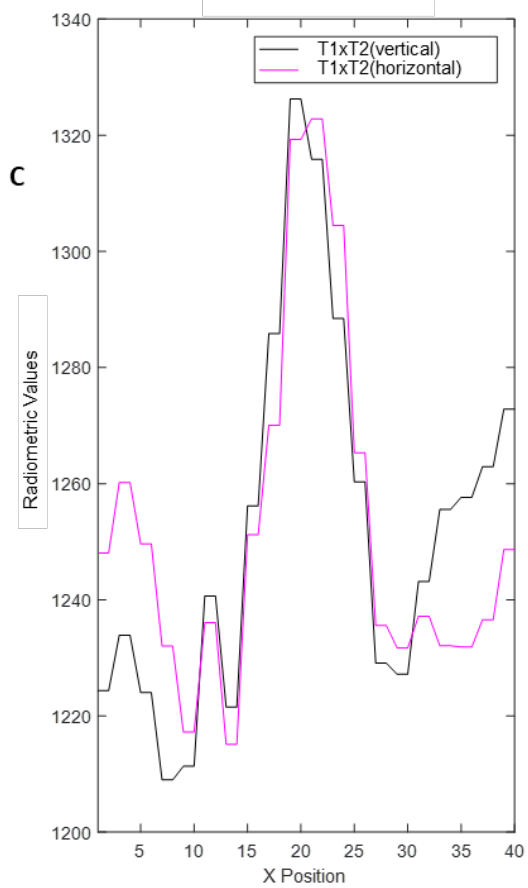

Fig. 2. Thermal cross-section profile methodology: $A$ - User selected $40 \times 40$ area; $B$ - Multiplication of crosssections of the diagonals over the normalized square; $\mathrm{C}$ - Characteristic thermal profiles.

\section{Results}

In total, 85 skin lesions were examined, being 35 Basal Cell Carcinomas (BCC), 26 Melanomas, 10 Melanocytic Nevus (MN), 7 Squamous Cell Carcinoma (SCC), 2 Seborrheic Keratosis (SK), 2 Actinic Keratosis (AK), 1 Atypical Nevus (AN), 1 Merkel Cell Carcinoma (MCC) and 1 Chondritis.

A clear separation between the average radiometric values of malignant and benign neoplasms was observed (Figure 3). Furthermore, the characteristic thermal profiles, for the same malignancy, were not significantly different.

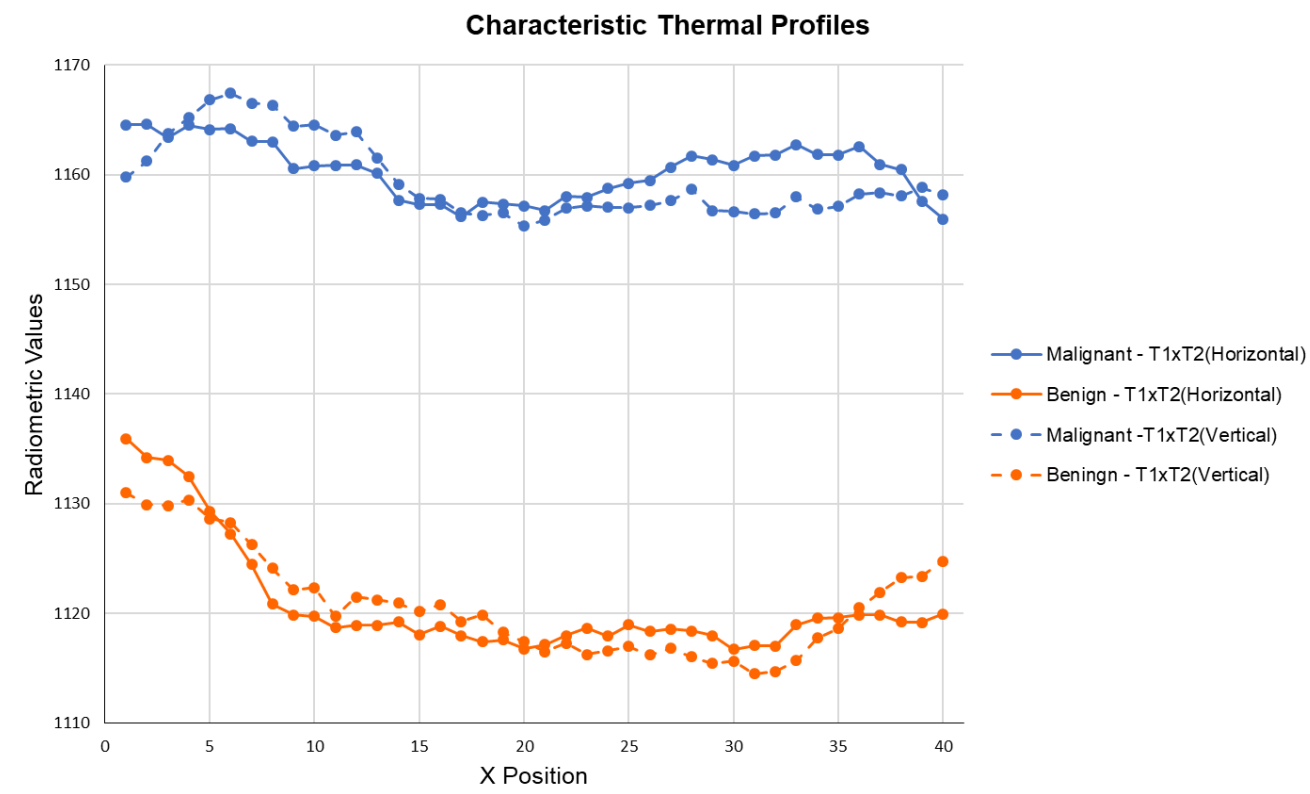

Fig. 3. Characteristic thermal profiles - T1XT2 (Horizontal) and T1XT2(Vertical) - for benign and malignant groups of neoplasia. 
The temperature profiles acquired for each skin neoplasm type were also fairly similar (Figure 4 and 5). The curves corresponding to malignant lesions, e.g., BCC, Melanoma, SCC and Merkel Cell Carcinoma, presented, in general, higher values than the benign ones. Only the radiometric values of AK and Seborrheic Keratosis samples exceed the ones of malignant neoplasia. Two temperature valleys were identified, one for SCC and another for Chondritis.

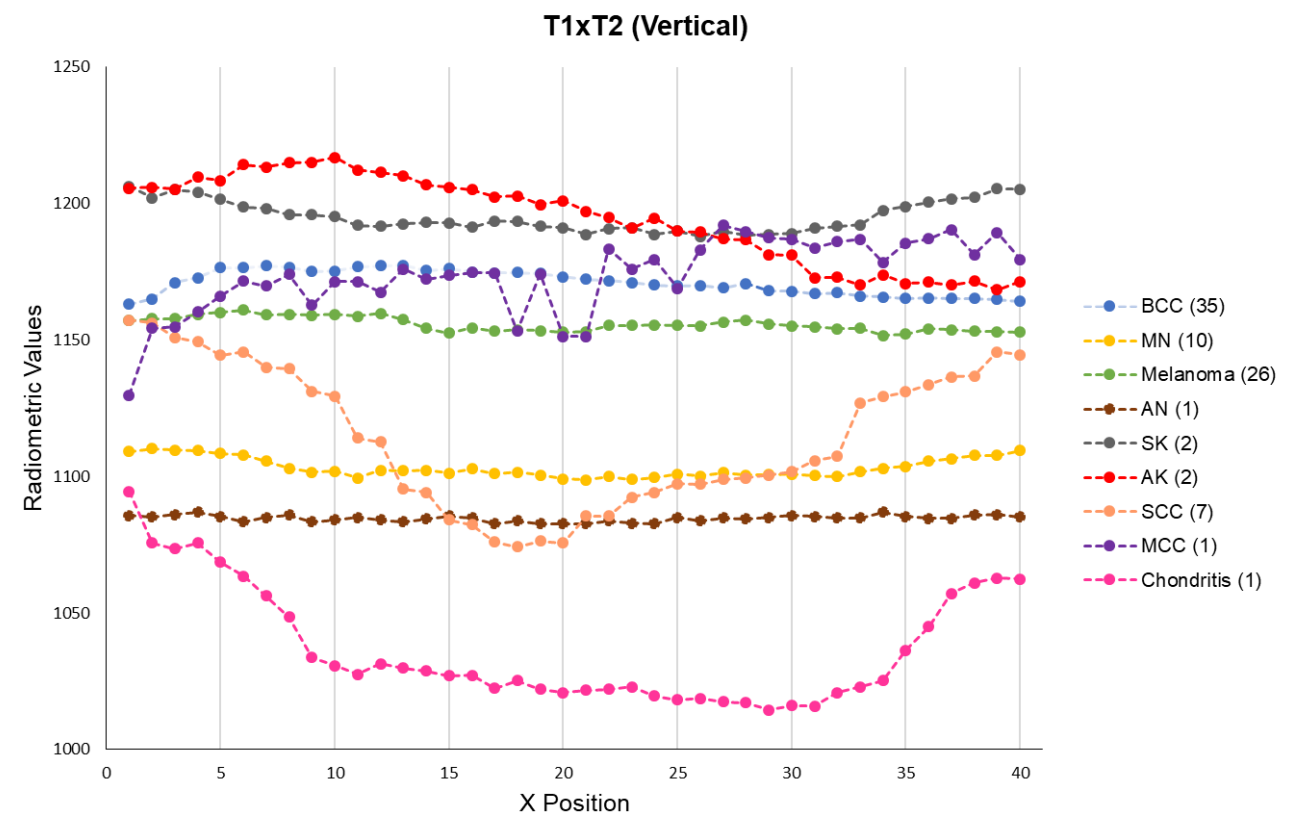

Fig. 4. Characteristic thermal profiles for each skin neoplasm type - T1xT2 (Vertical).

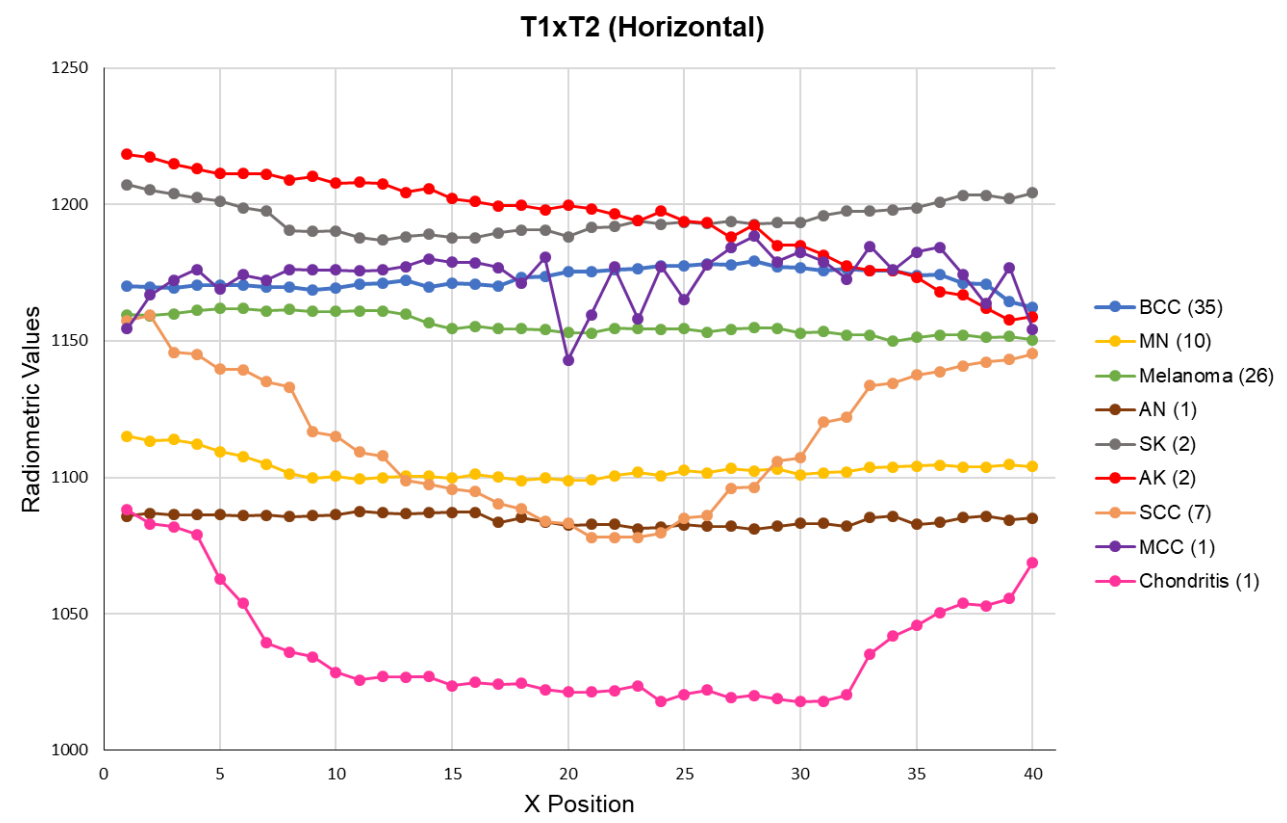

Fig. 5. Characteristic thermal profiles for each skin neoplasm type - T1xT2 (Horizontal).

The obtained classification accuracies are shown in table 1, from which it can be seen that the k-NN classifier was the classification method with best accuracy $(60 \%)$, followed by ANN (52\%) and the worst result was achieved by the SVN (44\%). 
Table 1. Results from the data classification accuracy at Weka.

\begin{tabular}{|l|c|c|c|}
\hline Classifiers & ANN & SVM & k-NN \\
\hline Accuracy & $52 \%$ & $44 \%$ & $60 \%$ \\
\hline
\end{tabular}

\section{Discussion}

The minor differences observed between the thermal curves T1xT2 (Vertical) and T1xT2 (Horizontal) suggest that either one of the profiles could be used for the identification and classification of skin lesions in thermographic studies (Figure 2, 3 and 4). As expected the curves obtained for malignant neoplasms showed, in general, higher temperature values when compared to the benign ones (Figure 3, 4 and 5). However, SCC, a malignant lesion, displayed a hypothermic pattern, instead of a hyperthermic one (Figure 4 and 5). This profile can be justified by the characteristic anatomy of this type of skin cancer, since a thick crust is commonly encountered on top of the lesion, acting as a thermal insulator. Furthermore, the profiles obtained for actinic and seborrheic keratosis - benign neoplasms - were higher than the malignant ones (Figure 4 and 5). Concerning the first, the explanation might be in the fact that this lesion type is usually portrayed as a pre-cancerous lesion, so it is possible that some of the actinic keratosis lesions were misdiagnosed, being in a more advanced state and having, therefore, higher temperature values. A hypothermic pattern should be characteristic of seborrheic keratosis' lesions. Thus, the contradictory outcome could only be explained by the lack of samples (two) for this type of lesion.

The obtained classification results were not very satisfactory. However, the number of participants enrolled is small and it is expected a rise in the accuracy with the increase of sample size. The enhancement of temperature differences between the lesion site and healthy skin might also contribute to better classification results.

\section{Conclusion}

The temperature values of thermal skin neoplasm images, acquired using steady-state thermography, were analyzed with a MATLAB application and used for statistical evaluation and sample classification.

The temperature profile of each skin tumor type was successfully obtained by either one of the main lesion diagonals, being as expected, except for SCC and actinic and seborrheic keratosis. The k-NN classifier was the one that attained the best classification results, even though unsatisfactory. The improvement of this results could be achieved with the increase of the number of samples included in the data set. For a future approach, instead of a static thermogram over the lesion, a cold thermal provocation stimulus with the analysis of its recovery might be more interesting. If successfully applied and with good classification results, it could, in most cases, provide a cheaper and faster result than biopsies and in an objective manner, contrasting with the dermatoscopy method.

\section{Acknowledgments}

The authors gratefully acknowledge the partial funding of project NORTE-01-0145-FEDER-000022 - SciTech Science and Technology for Competitive and Sustainable Industries, co-financed by Programa Operacional Regional do Norte (NORTE2020), through Fundo Europeu de Desenvolvimento Regional (FEDER) and of project LAETA UID/EMS/50022/2013.

\section{REFERENCES}

[1] J. Ferlay et al., "Cancer incidence and mortality patterns in Europe: Estimates for 40 countries in 2012," Eur. J. Cancer, vol. 49, no. 6, pp. 1374-1403, Apr. 2013.

[2] Linos E., S. SM., C. MG., C. GA., and C. CA., "Increasing burden of melanoma in the United States," J. Invest. Dermatol., vol. 129, no. 7, pp. 1666-74, 2009.

[3] D. A. Weinstein, S. Konda, and B. M. Coldiron, "Use of Skin Biopsies Among Dermatologists," Dermatologic Surg., vol. 43, no. 11, pp. 1348-1357, Nov. 2017.

[4] M. Pirtini Çetingül and C. Herman, "Quantification of the thermal signature of a melanoma lesion," Int. J. Therm. Sci., vol. 50, no. 4, pp. 421-431, Apr. 2011.

[5] I. A. Nola and D. Kolanc, "Thermography in biomedicine," in 201557 th International Symposium ELMAR (ELMAR), 2015, pp. 17-20.

[6] E. F. J. Ring and K. Ammer, "Infrared thermal imaging in medicine," Physiol. Meas., vol. 33, no. 3, pp. R33-R46, Mar. 2012.

[7] C. Magalhães, R. Vardasca, and J. Mendes, "Recent use of medical infrared thermography in skin neoplasms," Ski. Res. Technol., vol. 0, pp. 1-5, 2018.

[8] R. Vardasca, L. Vaz, and J. Mendes, "Classification and Decision Making of Medical Infrared Thermal Images," in 
Lecture Notes in Computational Vision and Biomechanics, vol. 26, 2018, pp. 79-104.

[9] K. Ammer and K. Ammer, "The Glamorgan Protocol for recording and evaluation of thermal images of the human body," Thermol. Int., vol. 18, no. 4, pp. 125-129, 2008.

[10] E. Ring and K. Ammer, "The technique of infrared imaging in medicine," in Infrared Imaging, IOP Publishing, 2015 , pp. $1-10$

[11] M. . Gardner and S. . Dorling, "Artificial neural networks (the multilayer perceptron)-a review of applications in the atmospheric sciences," Atmos. Environ., vol. 32, no. 14-15, pp. 2627-2636, 1998.

[12] L. J. Cao et al., "Parallel sequential minimal optimization for the training of support vector machines," IEEE Trans. Neural Networks, vol. 17, no. 4, pp. 1039-1049, 2006.

[13] C. Hulett, A. Hall, and G. Qu, "Dynamic Selection of k Nearest Neighbors in Instance-based Learning," pp. 85-92, 2012. 